\title{
COMPOSIÇÃO FLORÍSTICA E ESTRUTURA DA COMUNIDADE DE PLANTAS DO ESTRATO HERBÁCEO EM ÁREAS DE CULTIVO DE ÁRVORES FRUTÍFERAS ${ }^{1}$
}

\author{
YURIKO A. N. P. YANAGIZAWA² E RITA C. S. MAIMONI-RODELLA ${ }^{2}$
}

\section{RESUMO}

O objetivo do presente trabalho foi caracterizar, em três épocas, a comunidade de plantas presentes em áreas de cultivo de abacateiro e de nogueira pecan, localizadas na Fazenda Experimental Lageado, da FCA-UNESP, município de Botucatu-SP. Em decorrência da arquitetura da copa e do caráter decíduo das nogueiras, esta área apresenta-se mais ensolarada, o que produz condições ambientais diferentes entre as duas áreas estudadas. Foi estabelecida uma grade retangular composta de 55 parcelas quadradas, contíguas, com $5 \mathrm{~m}$ de lado, havendo 40 parcelas sob os abacateiros e 15 sob as nogueiras. As coletas foram realizadas em março, maio e agosto de 1993, quando foram sorteados quadrados de 1x $1 \mathrm{~m}$ em cada parcela, de forma a não haver coincidência entre as amostragens realizadas em cada época. Foram levantadas as espécies ocorrentes, sua porcentagem de cobertura e freqüência. Os dados foram analisados através de métodos multivariados, utilizando-se a Análise de Agrupamento para as seis situações (3 épocas x 2 ambientes). Foram coletadas, no total, 54 espécies, distribuídas em 38 gêneros e 19 famílias, sendo Asteraceae e Poaceae as que contribuíram com o maior número de espécies. A riqueza em espécies foi maior na área sob as nogueiras; em ambas as áreas, a riqueza foi maior na estação úmida. Sob os abacateiros, a porcentagem de cobertura total da comunidade apresentou pouca variação temporal, diferindo da área sob as nogueiras onde se observou flutuação deste parâmetro. O padrão de distribuição espacial, para quase todas as populações, foi do tipo agrupado. A similaridade florística foi maior entre as duas áreas dentro de cada época de coleta. A presença constante de espécies como Commelina nudiflora que contribuiu com valores elevados de cobertura nas três épocas de coleta, mostra a necessidade de utilizar métodos de controle permanentes na área do pomar estudado.

Palavras chave: Plantas daninhas, ecologia, pomar, abacateiro, nogueira pecan.

\section{ABSTRACT \\ Floristic composition and community structure of understory vegetation in orchard}

The main goal of this study was to describe the floristic composition and the community structure of the vegetation under avocado and pecan trees growing at Fazenda Experimental Lageado, FCA - UNESP, Botucatu SP, Brazil. The studied areas differ in relation to light exposure conditioned by canopy architecture and pecan leaf deciduousness. A rectangular frame measuring $25 \times 55 \mathrm{~m}$ was divided in 55 quadrats of $5 \times 5 \mathrm{~m}$. Forty quadrats were located under avocado trees and 15 under pecan trees. Frequency and percentage weed cover were estimated in both conditions at March, May and August 1993. Quadrats of $1 \times 1 \mathrm{~m}$ were

\footnotetext{
${ }^{1}$ Recebido para publicação em 24/11/98 e na forma revisada 07/10/99.

${ }^{2}$ Prof $^{\circ}$ Assistente Doutor, IBB/UNESP, Distrito de Rubião Jr. C.P. 510, CEP: 18618-000, Botucatu/SP.
} 
randomly assigned to a new area within each plot, each month. A Cluster Analysis was performed with six experimental groups (3 sampling dates $\mathrm{x}$ environmental conditions). Fifty four species, 38 genera and 19 families were recorded in total. Asteraceae and Poaceae had greater number of species. Species richness was greater at pecan tree community. In both areas, the richness was greater at the wet season. Total percentage of weed cover showed small variation only under the

\section{INTRODUÇÃO}

A caracterização de comunidades de plantas daninhas apresenta grande interesse por contribuir na detecção de problemas e na escolha de estratégias de manejo e de controle a serem empregadas, nas mais diversas condições de sistemas agrícolas.

Nos países da Europa e da América do Norte, levantamentos florísticos e caraterizações de comunidades daninhas vêm sendo desenvolvidos, podendo-se mencionar o trabalho de Streibig (1979), que discorreu sobre a importância de estudos quantitativos na análise de aspectos ecológicos e fitossociológicos, assim como os trabalhos de Chancellor (1985), Dale \& Thomas (1987), Salvá \& Bermejo (1988) que, através de métodos quantitativos evidenciaram uma série de correlações entre plantas daninhas e características do solo, do clima e das formas de manejo das culturas onde elas ocorrem. $\mathrm{O}$ conhecimento da comunidade de plantas daninhas tem importância fundamental na tomada de decisão referente a práticas de manejo e controle, nas mais diversas formas de cultivo, como tem sido evidenciado pelos trabalhos de Weber et al. (1995), Bárberi et al. (1997) e Stevenson et al. (1997).

No Brasil, esta linha de pesquisa é ainda incipiente. Podem-se citar alguns levantamentos realizados na cultura do milho (Fonseca, 1983), em áreas com diferentes tipos de solo (Pagliarini et al., 1993), em áreas de gramado (MaimoniRodella et al., 1993) e em diversas áreas de avocado trees during the study period. Most populations showed an aggregate distribution pattern. Floristic similarity was greater among the two areas at each sampling period. Commelina nudiflora and some other weed species had high cover values during all the study period. Therefore, a continuous weed control is indicated to the studied orchard.

Key words: Weeds, ecology, orchard, avocado, pecan.

cultura no estado do Paraná (Kranz, 1993). Nestes levantamentos, a flora ocorrente e os fatores determinantes das características observadas foram caracterizados. $\mathrm{O}$ presente trabalho foi realizado com o objetivo de caracterizar a comunidade de plantas ocorrentes no estrato herbáceo, em área de cultivo de árvores frutíferas, procurando avaliar a importância das condições ambientais sobre a composição florística e sobre a estrutura da comunidade vegetal ocorrente nesta área.

\section{MATERIAL E MÉTODOS}

O trabalho foi desenvolvido em áreas de cultivo de abacateiro e de nogueira pecan, localizadas na Fazenda Experimental Lageado, da Faculdade de Ciências Agronômicas - UNESP, município de Botucatu, SP (22 $52^{\circ} 20^{\prime \prime} \mathrm{S}$ $48^{\circ} .26^{\prime} 37^{\prime \prime}$ WGW). O local de estudo situa-se a cerca de $800 \mathrm{~m}$ de altitude e apresenta solo classificado como Terra Roxa Estruturada, Textura Argilosa; o clima da região, segundo o sistema de Koeppen, é mesotérmico de inverno seco (Cwb), sendo julho o mês mais seco e frio e janeiro o mês mais quente e úmido (Carvalho et al. , 1983).

Para o levantamento da comunidade de plantas daninhas ocorrentes no local, foram escolhidas duas áreas contíguas, sendo uma delas localizada sob as copas de árvores adultas de abacateiro (Persea americana) e a outra, sob as copas de árvores adultas de 
nogueira pecan (Carya illinoensis). Foi estabelecida uma grade retangular composta de 55 parcelas quadradas, contíguas, com $5 \mathrm{~m}$ de lado, havendo 40 parcelas sob os abacateiros e 15 sob as nogueiras. Em decorrência da diferente arquitetura das copas apresentada pelas árvores frutíferas, ou seja copa com ramificação vertical no caso das nogueiras e copa com ramificação horizontal, no caso dos abacateiros, a primeira apresenta-se mais ensolarada. Além disso, devido ao caráter decíduo das nogueiras, esta área permanece totalmente exposta ao sol nos meses de seca, o que acentua as diferenças nas condições ambientais em relação à área sob os abacateiros, que são perenes. Foram realizadas coletas em três épocas - março, maio e agosto de 1993, de forma a abranger períodos com diferentes condições climáticas.

Em cada coleta, foram sorteados quadrados de $1 \times 1 \mathrm{~m}$ em cada parcela, de forma a não haver coincidência entre os quadrados amostrados em cada época. Foram levantadas as espécies ocorrentes, sua porcentagem de cobertura e frequiência. Os dados foram analisados por meio de métodos multivariados, utilizando-se de Análise de Agrupamento para as seis situações - 3 épocas x 2 áreas - com base na Distância Euclidiana Média (Sneath \& Sokal, 1973). Os resultados foram organizados em dendrogramas onde a abscissa indica o nível de similaridade entre as seis situações analisadas. A riqueza em espécies em cada área foi estimada pela razão número total de espécies/área, padronizando-se esta estimativa para $15 \mathrm{~m}^{2}$. O padrão de distribuição espacial das populações foi calculado por meio do Índice de Morisita Padronizado (Krebs, 1989).

\section{RESULTADOS E DISCUSSÃO}

Foram coletadas, no total, 54 espécies distribuídas em 38 gêneros e 19 famílias (Tabela 1). Deste total, 44 espécies são dicotiledôneas e 10 são monocotiledôneas. Asteraceae (=Compositae) e Poaceae (=Gramineae) são as famílias que contribuíram com maior número de espécies, sendo ambas geralmente apontadas como as mais representativas em comunidades de plantas daninhas, em diferentes regiões (Fonseca et al., 1983; Salva \& Bermejo, 1988; Hidalgo et al., 1990; Weber et al., 1995; Stevenson et al., 1997). Cerca de $80 \%$ das espécies são herbáceas e o restante são arbustivas ou subarbustivas. Em relação ao ciclo, $53 \%$ são espécies perenes e o restante, espécies anuais. Esta proporção de espécies perenes na área de estudo demonstra que seu estabelecimento é favorecido em áreas com menores níveis de alteração antrópica, como é o caso de áreas de cultivo de plantas frutíferas. Em ambientes extremamente variáveis nos quais fatores como a umidade, luz e temperatura oscilam intensamente, a presença de plantas daninhas anuais é predominante (Bazzaz \& Morse, 1991).

As áreas sob os abacateiros $\left(40 \mathrm{~m}^{2}\right)$ e sob as nogueiras $\left(15 \mathrm{~m}^{2}\right)$, apresentaram variações quanto à composição florística, observando-se a ocorrência de maior número de espécies na área sob os abacateiros. Este resultado pode estar associado ao tamanho da área pois o número de espécies tende, em princípio, a aumentar com o incremento da área amostrada (Mueller-Dombois \& Ellenberg, 19745; Kershall \& Looney, 1985). Das 36 espécies inicialmente encontradas sob os abacateiros (março), 19 ocorreram também no mês de maio e 18 no mês de agosto; 6 e 3 espécies diferentes surgiram nas duas últimas coletas, respectivamente. Sob as nogueiras, das 26 espécies iniciais ocorreram apenas 9 no mês de maio e 11 no mês de agosto, havendo, respectivamente, surgimento de 2 e 6 outras espécies.

A Análise de Agrupamento, quanto à composição florística, evidenciou maior similaridade entre as áreas sob abacateiros e sob nogueiras em março (Grupo 1), contra as demais situações (Grupo 2: maio e agosto), conforme apresentada na Figura 1. Este resultado sugere que a variação estacional interfere mais acentuadamente sobre a composição florística do que as diferenças existentes entre as duas situações estudadas. 
TABELA 1. Espécies ocorrentes no estrato herbáceo em áreas de cultivo de abacateiro e nogueira pecan, na Fazenda Experimental Lageado - UNESP, Botucatu, SP

\begin{tabular}{|c|c|c|c|c|c|c|c|c|c|c|c|c|}
\hline \multirow{3}{*}{ FAMÍLIA / ESPÉCIE } & \multicolumn{6}{|c|}{ ABACATEIRO } & \multicolumn{6}{|c|}{ NOGUEIRA PECAN } \\
\hline & \multicolumn{2}{|c|}{ MARÇO } & \multicolumn{2}{|c|}{ MAIO } & \multicolumn{2}{|c|}{ AGOSTO } & \multicolumn{2}{|c|}{ MARÇO } & \multicolumn{2}{|c|}{ MAIO } & \multicolumn{2}{|c|}{ AGOSTO } \\
\hline & Cob. & Freq. & Cob. & Freq. & Cob. & Freq. & Cob. & Freq. & Cob. & Freq. & Cob. & Freq. \\
\hline $\begin{array}{r}\text { Apocynaceae } \\
\text { 1. Peschiera fuchsiaefolia } \\
\text { Asteraceae }\end{array}$ & 1,80 & 22,50 & 0,38 & 5,00 & 0,80 & 12,50 & 0,80 & 40,00 & 0,47 & 13,33 & 0,13 & 6,67 \\
\hline 2. Acanthospermum australe & - & - & - & - & - & - & 0,13 & 6,67 & - & - & - & - \\
\hline 3. Baccharis sp. & - & - & - & - & - & - & 0,33 & 6,67 & - & - & - & - \\
\hline 4. Bidens pilosa & 0,10 & 5,00 & 0,05 & 2,50 & 0,28 & 7,50 & - & - & - & - & - & - \\
\hline 5. Chaptalia integerrima & - & - & - & - & - & - & 0,07 & 6,67 & 0,33 & 6,67 & - & - \\
\hline 6. Chaptalia nutans & 0,10 & 2,50 & - & - & - & - & - & - & - & - & 0,47 & 6,67 \\
\hline 7. Emilia sonchifolia & - & - & 0,03 & 2,50 & 0,50 & 5,00 & 0,07 & 6,67 & - & - & 1,00 & 6,67 \\
\hline 8. Erechtites valerianaefolia & 0,25 & 7,50 & 0,63 & 5,00 & - & - & 0,33 & 6,67 & - & - & - & - \\
\hline 9. Eupatorium sp. & 0,08 & 2,50 & - & - & - & - & 0,07 & 6,67 & - & - & - & - \\
\hline 10. Facelis retusa & - & - & - & - & 0,10 & 2,50 & - & - & - & - & - & - \\
\hline 11. Galinsoga parviflora & - & - & 0,05 & 2,50 & - & - & - & - & - & - & - & - \\
\hline 12. Gamochaeta americana & 0,05 & 5,00 & - & - & 0,05 & 2,50 & 0,33 & 6,67 & - & - & - & - \\
\hline 13. Gamochaeta pensilvanica & - & - & - & - & - & - & - & - & - & - & 0,13 & 6,67 \\
\hline 14. Gamochaeta sp & 0,03 & 2,50 & 0,05 & 2,50 & - & - & - & - & - & - & - & - \\
\hline 15. Mikania scandens & 0,93 & 10,00 & - & - & - & - & 1,00 & 6,67 & - & - & - & - \\
\hline 16. Senecio brasiliensis & 0,13 & 2,50 & 0,25 & 2,50 & - & - & - & - & - & - & - & - \\
\hline 17. Vernonia polyanthes & - & - & 0,88 & 2,50 & - & - & - & - & - & - & - & - \\
\hline 18. Vernonia sp. & - & - & - & - & - & - & - & - & - & - & 0,33 & 6,67 \\
\hline Caryophylaceae & & & & & & & & & & & & \\
\hline 19. Drymaria cordata & - & - & 0,05 & 2,50 & - & - & - & - & - & - & - & - \\
\hline $\begin{array}{l}\text { 20. Drymaria sp. } \\
\text { Commelinaceae }\end{array}$ & - & - & - & - & 0,03 & 2,50 & - & - & - & - & - & - \\
\hline $\begin{array}{l}\text { 21. Commelina nudiflora } \\
\text { Convolvulaceae }\end{array}$ & 10,63 & 47,50 & 16,88 & 52,50 & 12,80 & 57,50 & 0,07 & 6,67 & 0,33 & 6,67 & 1,33 & 6,67 \\
\hline $\begin{array}{l}\text { 22. Ipomoea cairica } \\
\text { Cyperaceae }\end{array}$ & 0,03 & 2,50 & 0,13 & 2,50 & - & - & - & - & - & - & - & - \\
\hline 23. Cyperus brevifolius. & 0,05 & 2,50 & - & - & - & - & - & - & - & - & - & - \\
\hline $\begin{array}{l}\text { 24. Cyperus iria } \\
\text { Euphorbiaceae }\end{array}$ & - & - & - & - & - & - & - & - & - & - & 0,07 & 6,67 \\
\hline 25. Euphorbia heterophylla & 0,05 & 5,00 & - & - & - & - & 0,13 & 6,67 & - & - & - & - \\
\hline 26. Phyllanthus corcovadensis & 0,43 & 15,00 & - & - & 0,40 & 12,50 & 0,67 & 13,33 & & & 0,13 & 6,67 \\
\hline $\begin{array}{l}\text { 27. Phyllanthus sp. } \\
\text { Lamiaceae }\end{array}$ & 0,50 & 15,00 & 0,35 & 12,50 & - & - & 0,40 & 13,33 & 0,67 & 13,33 & - & - \\
\hline 28. Hyptis suaveolens & 0,10 & 5,00 & - & - & 0,58 & 5,00 & - & - & - & - & - & - \\
\hline
\end{tabular}

continua.

TABELA 1. Continuação... 


\begin{tabular}{|c|c|c|c|c|c|c|c|c|c|c|c|c|}
\hline \multirow{3}{*}{ FAMÍLIA / ESPÉCIE } & \multicolumn{6}{|c|}{ ABACATEIRO } & \multicolumn{6}{|c|}{ NOGUEIRA PECAN } \\
\hline & \multicolumn{2}{|c|}{ MARÇO } & \multicolumn{2}{|c|}{ MAIO } & \multicolumn{2}{|c|}{ AGOSTO } & \multicolumn{2}{|c|}{ MARÇO } & \multicolumn{2}{|c|}{ MAIO } & \multicolumn{2}{|c|}{ AGOSTO } \\
\hline & Cob. & Freq. & Cob. & Freq. & Cob. & Freq. & Cob. & Freq. & Cob. & Freq. & Cob. & Freq. \\
\hline Malvaceae & & & & & & & & & & & & \\
\hline 29. Sida glaziovii & 0,68 & 15,00 & 0,63 & 12,50 & - & - & 0,13 & 13,33 & - & - & 1,00 & 6,67 \\
\hline 30. Sida rhombifolia . & 0,30 & 5,00 & - & - & 0,30 & 10,00 & 0,13 & 6,67 & - & - & 0,13 & 13,33 \\
\hline 31. Sida santaremnensis & 0,03 & 2,50 & - & - & - & - & 0,33 & 6,67 & - & - & - & - \\
\hline 32. Sida sp. & 0,15 & 5,00 & - & - & - & - & - & - & - & - & - & - \\
\hline $\begin{array}{l}\text { Oxalidaceae } \\
\text { 33. Oxalis martiana } \\
\text { Piperaceae }\end{array}$ & - & - & 0,08 & 5,00 & - & - & - & - & - & - & - & - \\
\hline 34Piper sp. & - & - & 0,75 & 2,50 & 0,25 & 2,50 & - & - & - & - & - & - \\
\hline $\begin{array}{l}\text { Poaceae } \\
\text { 35. Brachiaria decumbens }\end{array}$ & 9,60 & 40,00 & 7,25 & 32,50 & 1,13 & 10,00 & 7,87 & 33,33 & 9,13 & 40,00 & 7,53 & 33,33 \\
\hline $\begin{array}{c}\text { 36. Digitaria insularis } \\
\text { Poaceae }\end{array}$ & 0,48 & 7,50 & - & - & 0,25 & 2,50 & 0,87 & 13,33 & 1,33 & 6,67 & - & - \\
\hline 37. Panicum maximum & 0,13 & 5,00 & 0,43 & 7,50 & 3,80 & 22,50 & 1,60 & 13,33 & 5,13 & 20,00 & 12,67 & 33,33 \\
\hline 38. Paspalum conspersum & 1,15 & 7,50 & - & - & - & - & 3,87 & 20,00 & - & - & - & - \\
\hline 39. Paspalum dilatatum & - & - & - & - & - & - & 1,33 & 6,67 & - & - & - & - \\
\hline 40. Paspalum sp. & - & - & - & - & 0,50 & 7,50 & - & - & - & - & 0,67 & 6,67 \\
\hline $\begin{array}{l}\text { 41. Pennisetum purpureum } \\
\text { Polygonaceae }\end{array}$ & 0,03 & 2,50 & 1,25 & 2,50 & - & - & - & - & - & - & - & - \\
\hline $\begin{array}{l}\text { 42.Polygonum sp. } \\
\text { Portulacaceae }\end{array}$ & 0,03 & 2,50 & - & - & - & - & - & - & - & - & - & - \\
\hline $\begin{array}{l}\text { 43.Tallinum patens } \\
\text { Rosaceae }\end{array}$ & 5,43 & 72,50 & 5,45 & 62,50 & 10,73 & 55,00 & 5,40 & 60,00 & 0,80 & 13,33 & 1,33 & 6,67 \\
\hline $\begin{array}{l}\text { 44. Rubus brasiliensis } \\
\text { Rubiaceae }\end{array}$ & 0,03 & 2,50 & - & - & - & - & - & - & - & - & - & - \\
\hline 45.Borreria alata & - & - & 0,13 & 2,50 & - & - & - & - & - & - & - & - \\
\hline $\begin{array}{r}\text { 46.Richardia brasiliensis } \\
\text { Solanaceae }\end{array}$ & 0,03 & 2,50 & 0,18 & 5,00 & 2,08 & 7,50 & - & - & 0,33 & 6,67 & - & - \\
\hline 47. Solanum americanum & - & - & 0,25 & 5,00 & - & - & - & - & - & - & 0,20 & 6,67 \\
\hline 48.Solanum erianthum & 0,13 & 2,50 & 0,38 & 7,50 & 0,83 & 10,00 & & & 0,67 & 6,67 & & \\
\hline 49. Solanum paniculatum & 0,45 & 5,00 & - & - & - & - & 0,20 & 6,67 & - & - & - & - \\
\hline 50. Solanum sp. 1 & 1,25 & 20,00 & 0,05 & 2,50 & 0,25 & 5,00 & 0,60 & 20,00 & - & - & 1,33 & 13,33 \\
\hline 51. Solanum sp. 2 & 0,05 & 5,00 & - & - & - & - & - & - & - & - & - & - \\
\hline $\begin{array}{l}\text { Tiliaceae } \\
\text { 52. Triumfetta semitriloba } \\
\text { Verbenaceae }\end{array}$ & 0,20 & 7,50 & 0,25 & 2,50 & 2,05 & 7,50 & 0,47 & 6,67 & - & - & - & - \\
\hline $\begin{array}{l}\text { 53. Aegiphila sellowiana } \\
\text { 54. Lantana camara }\end{array}$ & $\begin{array}{l}1,25 \\
0,08 \\
\end{array}$ & $\begin{array}{l}2,50 \\
5,00 \\
\end{array}$ & - & - & 0,38 & 5,00 & 0,93 & 13,33 & 0,33 & 6,67 & 0,33 & 6,67 \\
\hline TOTAL & 36,74 & - & 36,81 & - & 38,09 & - & 28,13 & - & 19,52 & - & 28,78 & - \\
\hline
\end{tabular}


A riqueza em espécies, que expressa a razão entre o número de espécies e a área levantada foi, em geral, maior na área sob as nogueiras (Tabela 2), o que pode ser decorrência da maior incidência de luz neste local, favorecendo o estabelecimento de plantas daninhas que são na maioria, heliófitas (Leitão Filho et al., 1972; 1975). Para cada área, a riqueza específica foi maior na estação úmida (março) do que na seca (maio e agosto), indicando que muitas das espécies finalizam o seu ciclo na estação seca.

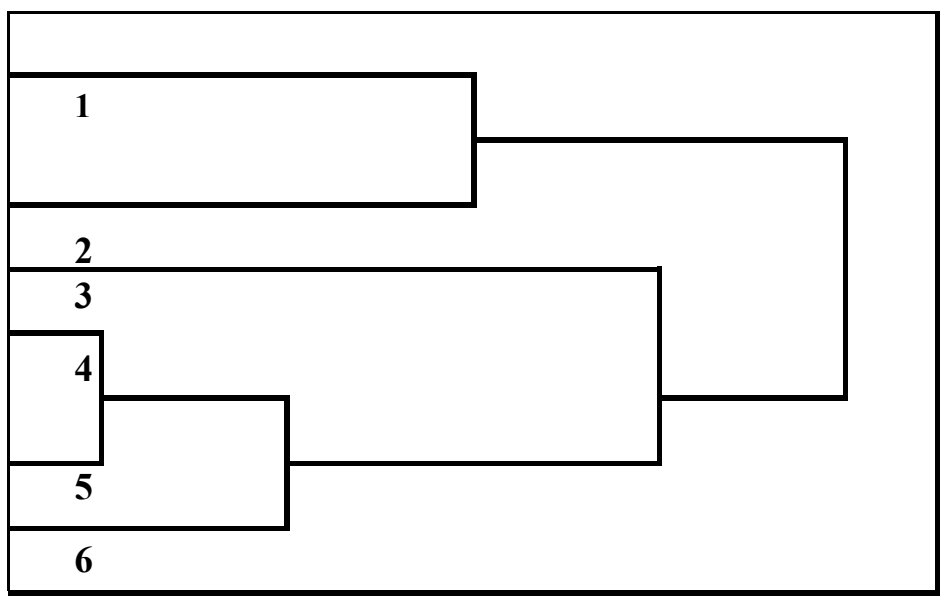

FIGURA 1. Dendrograma resultante da Análise de Agrupamento utilizando-se a Distância Euclidiana Média para os dados de Presença/Ausência dos componentes de duas comunidades de plantas daninhas, avaliadas em três épocas distintas. 1-3: sob abacateiros, em março, maio e agosto. 4-6: sob nogueiras, em março, maio e agosto.

TABELA 2. Riqueza em espécies em comunidade do estrato herbáceo em áreas de pomar, Fazenda Experimental Lageado, UNESP, Botucatu, SP.

\begin{tabular}{|c|c|c|c|c|c|}
\hline \multicolumn{3}{|c|}{ ABACATEIRO } & \multicolumn{3}{|c|}{ NOGUEIRA PECAN } \\
\hline MARÇO & MAIO & AGOSTO & MARÇO & MAIO & AGOSTO \\
\hline 1,50 & 1,26 & 0,60 & 1,73 & 0,73 & 1,13 \\
\hline
\end{tabular}

A Tabela 1 apresenta dados sobre a porcentagem de cobertura obtida para as comunidades. Verifica-se que, sob os abacateiros, a porcentagem de cobertura total apresentou pouca variação temporal. Entre os componentes desta comunidade, várias espécies ocorreram apenas na época chuvosa. Desta forma, a manutenção da porcentagem de cobertura total na época seca deveu-se à manutenção e/ou ao aumento da cobertura por espécies remanescentes como Commelina nudiflora, Tallinum patens e
Pennisetum purpureum. Muitas das populações que ocorreram apenas na primeira coleta apresentaram baixos valores de cobertura, não contribuindo de forma relevante para o parâmetro em questão. Considerando-se que a cobertura pode expressar o grau de dominância da espécie na comunidade (Braun-Blanquet, 1979), C. nudiflora, T. patens e Brachiaria decumbens foram, no conjunto, as espécies mais 
dominantes na área sob os abacateiros.

No caso da comunidade sob as nogueiras, a flutuação da porcentagem de cobertura total da comunidade foi mais acentuada (Tabela 2). Muitas populações não foram amostradas a partir da segunda coleta; das remanescentes, a maioria apresentou redução e, embora algumas tenham aumentado a porcentagem de cobertura, no conjunto, o valor deste parâmetro diminuiu. $\mathrm{Na}$ terceira coleta, a porcentagem de cobertura total aumentou, sendo ligeiramente maior que a obtida na primeira coleta. As espécies dominantes neste período foram Brachiaria decumbens, T. patens e Paspalum conspersum; na segunda e terceira coletas, B. decumbens e Panicum maximum foram as dominantes.

O resultado da Análise de Agrupamento para a cobertura evidenciou que as três coletas realizadas sob as nogueiras foram bastante similares, constituindo um grupo (Grupo 1). Para a comunidade sob os abacateiros, houve o agrupamento entre as três coletas (Grupo 2), porém o nível de similaridade foi menor (Figura 2).

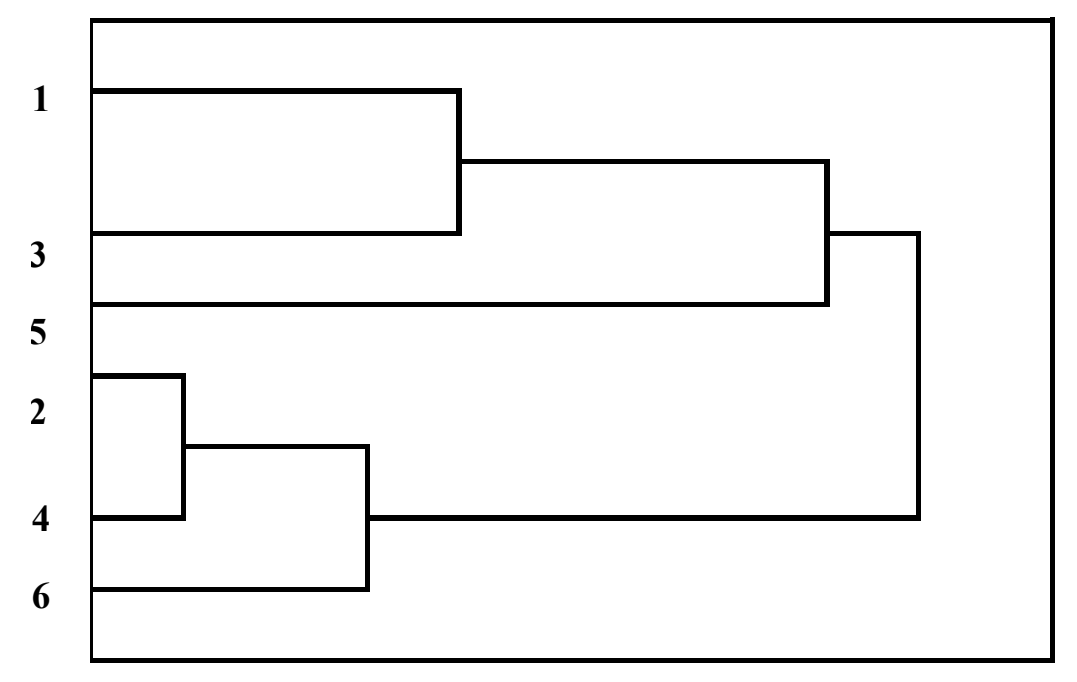

FIGURA 2. Dendrograma resultante da Análise de Agrupamento utilizando-se a Distância Euclidiana Média para os dados de Cobertura dos componentes de duas comunidades de plantas daninhas, avaliadas em três épocas distintas. 1-3: sob abacateiros, em março, maio e agosto. 4-6: sob nogueiras, em março, maio e agosto.

Os resultados sobre a freqüência das espécies estão apresentados na Tabela 3. A frequiência é uma estimativa da probabilidade de se encontrar uma espécie numa unidade de amostragem, sendo influenciada pela densidade e pelo grau de agrupamento dos indivíduos conspecíficos (Mueller-Dombois \& Ellenberg, 1974). As espécies T. patens, C. nudiflora, $B$. decumbens, Peschiera fuchsiaefolia e P. maximum foram as mais frequientes em ambas as áreas, porém as duas primeiras apresentaram maiores valores deste parâmetro na área sob os abacateiros, enquanto as demais foram mais freqüentes na área sob as nogueiras. Sendo $T$. patens e $C$. nudiflora plantas associadas preferencialmente a lugares sombreados (Leitão Filho et al., 1972; 1975; Bacchi et al., 1984), os resultados ora obtidos para frequiência e cobertura estão em conformidade com as suas exigências ecológicas. A deciduidade das nogueiras, permitindo maior exposição do substrato à luz, pode ser um fator que condiciona 
o recrutamento e/ou o crescimento de plantas como $P$. fuchsiaefolia, $B$. decumbens e $P$. maximum, que ocorrem caracteristicamente em áreas abertas e ensolaradas (Lorenzi, 1982), uma vez que estas espécies foram mais freqüentes e dominantes nesta área.

O resultado da Análise de Agrupamento para a freqüência evidenciou que as duas últimas coletas realizadas sob as nogueiras foram bastante similares, constituindo um grupo (Grupo1). A primeira coleta sob as nogueiras agrupou-se com as três coletas sob os abacateiros, apresentando os componentes deste grupo (Grupo 2), menores níveis de similaridade. Isto indica que, com o passar do tempo, ocorreu uma gradativa alteração da freqüência sob os abacateiros de forma a se assemelhar à freqüência obtida para a primeira coleta sob as nogueiras (Figura 3).

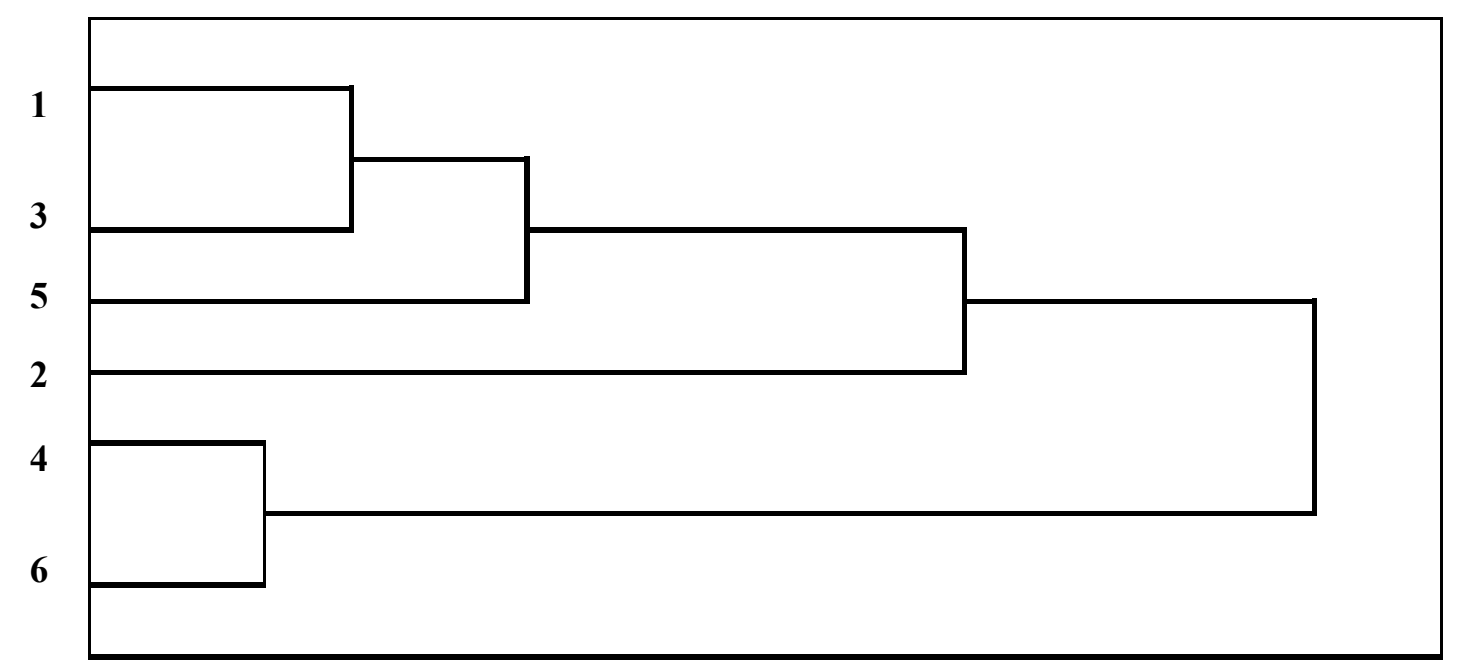

FIGURA 3. Dendrograma resultante da Análise de Agrupamento utilizando-se a Distância Euclidiana Média para os dados de Frequiência dos componentes de duas comunidades de plantas daninhas, avaliadas em três épocas distintas. 1-3: sob abacateiros, em março, maio e agosto. 46: sob nogueiras, em março, maio e agosto.

O padrão de distribuição espacial para as populações das duas comunidades foi, na sua quase totalidade, do tipo agrupado (cerca de $97 \%$ das espécies), o que pode ser indício da distribuição em mosaico dos recursos e condições ambientais favoráveis a cada espécie. Os resultados sobre a ocorrência de distribuição aleatória em algumas populações (cerca de 3\%), podem ser fruto de um artifício de amostragem, uma vez que foram obtidos com um número muito baixo de amostras, em decorrência da baixa densidade observada nestas populações. Embora os métodos para a avaliação do padrão de distribuição espacial das populações ainda sejam objeto de controvérsia, sabe-se que, na prática, as populações de todas as espécies estão distribuídas em mosaico (Begon et al., 1996) e esta distribuição é, em grande parte, condicionada pela heterogeneidade ambiental que é freqüentemente encontrada na natureza (Novoplansky, 1996; Fitter, 1997). No ambiente das comunidades ora estudadas, esta heterogeneidade influenciou a distribuição espacial obtida.

Os resultados ora levantados evidenciam o caráter altamente dinâmico de populações de plantas daninhas, expresso por marcantes alterações na estrutura entre duas comunidades estabelecidas em áreas que, embora contíguas, apresentam diferentes características ambientais. 
A diferença entre as áreas foi certamente influenciada pelas característica da arquitetura das copas e da deciduidade de cada espécie de árvore frutífera.

A pequena variação temporal nos valores de cobertura total, para ambas as áreas, evidencia a necessidade de realizar controle contínuo de plantas daninhas. As espécies que se mostraram dominantes nas condições do presente estudo são referidas como bastante agressivas em áreas de pastagens, culturas anuais e perenes e áreas ruderais (Lorenzi, 1982). C. nudiflora, que apresentou altos valores de cobertura e frequiência, merece destaque pois muitas espécies da família Commelinaceae vêm se tornando, no Brasil, um grave problema em áreas de culturas perenes (Kissmann, 1997), devido à sua resistência aos métodos de controle mecânico e químico, atualmente disponíveis (Velini").

\section{AGRADECIMENTOS}

Agradecemos ao Clemente José Campos, funcionário do Departamento de Botânica, Instituto de Boiciências, UNESP, Campus de Botucatu e à bióloga Cláudia Maria Liba pelo auxílio no trabalho de campo.

\section{LITERATURA CITADA}

BACCHI, O., LEITÃO FILHO, H. F., ARANHA, C. Plantas invasoras de culturas no estado de São Paulo. Campinas: UNICAMP, 1984. v.3, p. 598-906.

BÁRBERI，P., SILVESTRI，N., BONARI，E. Weed communities of winter as influenced by imput level and rotation. Weed Res., v. 37, n.5, p.301-13, 1997.

BAZZAZ, F.A.; MORSE, S.R. Annual plants: potential responses to multiple stresses. In: MOONEY, H.A; WINNER, W.E.; PELL,

\footnotetext{
"VELINI, E. D. (Faculdade de Ciências Agronômicas, UNESP - Campus de Botucatu). Comunicação pessoal, 1998.
}

E.J. (ed.). Responses of plant to multiple stresses. New York: Academic Press, 1991. cap. 13, p. 283-305.

BEGON, M., HARPER, J.L., TOWNSEND, C.R. Ecology: individuals, populations and communities. 3ed. Oxford: Blackwell Science, 1996. p.174.

BRAUN-BLANQUET, J. Fitosociologia: Bases para el estudio de las comunidades vegetales. Madrid: H. Blume, 1979. p. 2264.

CARVALHO, W. A., ESPINDOLA, C.R., PACCOLA, A.A. Levantamento de solos da Fazenda Lageado. Botucatu: FCAUNESP, 1983. (Boletim Científico,1)

CHANCELOR, R. J. Changes in weed flora of an arable field cultivated for 20 years. J. Appl. Ecol., v.22, p. 491-502, 1985.

DALE, M.R.T., THOMAS, A.G. The structure of weed communities in Saskatchewan fields. Weed Sci., v.35, p.348-55, 1987.

FITTER, A. Nutrient acquisition. In: CRAWLEY, M.J. (ed.). Plant ecology. 2 ed., Oxford: Blackwell Science, 1997. cap. 3, p. 51-72.

FONSECA, M.A., SALOMÃO, T. A., VICTORIA FILHO, R., ARANHA, C. Plantas daninhas na cultura de milho (Zea mays L.) na região de Jaboticabal - SP. Científica, v.11, p.279-87, 1983.

HIDALGO, B., SAAVEDRA, M., GARCIATORRES, L. Weed flora of dryland crops in the Cordoba region (Spain). Weed Res., v.30, p.309-18, 1990. 
KERSHALL, K. A., LOONEY, J. H. H. Quantitative and dynamic plant ecology. 3ed. London: Edward Arnold, 1985. p. 156-175.

KISSMAN, K. G. Plantas infestantes e nocivas. 2ed. São Paulo: BASF, 1997. v.1, p. 96-119.

KRANZ, W.M. Diagnóstico das plantas invasoras nos sistemas de manejo de solos do estado do Paraná. In: CONGRESSO BRASILEIRO DE HERBICIDAS E PLANTAS DANINHAS, 19, 1993. Resumos... Londrina: SBHED, 1993. p. 82-83.

KREBS, C.H.J. Ecological methodology. New York: Harper \& Row, 1989. cap.4, p.150154.

LEITÃO FILHO, H. F., ARANHA, C., BACCHI, O. Plantas invasoras de culturas no estado de São Paulo. São Paulo: HUCITEC, 1972. v.1, p. 1-291.

LEITÃO FILHO, H. F., ARANHA, C., BACCHI, O. Plantas invasoras de culturas no estado de São Paulo. São Paulo: HUCITEC/AGIPLAN, 1975. v.2, p. 292597.

LORENZI, H. Plantas daninhas do Brasil: terrestres, aquáticas, parasitas, tóxicas e medicinais. 2ed. Nova Odessa, SP: Editora Plantarum, 1991. $440 \mathrm{p}$.

MAIMONI-RODELLA, R.C.S., SILVA, R.C.B., RODELLA, R.A., CAMPOS, C.J. Levantamento da comunidade de plantas daninhas ocorrentes em áreas de gramado em Botucatu-SP. In: CONGRESSO BRASILEIRO DE HERBICIDAS E PLANTAS DANINHAS, 19, 1993. Resumos... Londrina: SBHED, 1993. p. 79.

MUELLER-DOMBOIS, D., ELLENBERG, H. Aims and methods of vegetation ecology.
New York: John Wiley \& Sons, 1974. p. 45-66.

NOVOPLANSKY, A. Developmental responses of individual Onobrychis plants to spatial heterogeneity. Vegetatio, v.127, p.31-9, 1996.

PAGLIARINI, A.C., MAIMONI-RODELLA, R.C.S., CURI, P. R. Composição florística de comunidades de plantas daninhas ocorrentes em Botucatu-SP. In: CONGRESSO BRASILEIRO DE HERBICIDAS E PLANTAS DANINHAS, 19, 1993. Resumos... Londrina: SBHED, 1993. p. 79-80.

SALVÁ, A. P., BERMEJO, J. E. H. Floristic composition and agricultural importance of weeds in southern Spain. Weed Res., v.28, p.175-80, 1988.

SNEATH, P.H.A., SOKAL, R. R. Numerical Taxonomy. San Francisco: W. H. Freeman, 1973. 573p.

STEVENSON, F.C., LEGERE, A., SIMARD, R.R., ANGERS, D.A., PAGEAU, D.; LAFOND, J. Weed species diversity in spring barley varie with crop rotation and tillsage, but not with nutrient source. Seed Sc., v.45, p.798-806, 1997.

STREIBIG, J.C. Numerical methods illustrating the phytosociology of crops in relation to weed flora. J. Appl. Ecol., v.16, p.577-87, 1979.

WEBER, G., ELEMO, K., LAGOKE, S.T.O. Weed communities in intensified cerealbased croping systems of the northern Guinea savanna. Weed Res., v.35, p.16778, 1995. 